\title{
Platelet-derived Growth Factor Receptors on Macrovascular Endothelial Cells Mediate Relaxation via Nitric Oxide in Rat Aorta
}

\author{
Leslie D. Cunningham, Peter Brecher, and Richard A. Cohen \\ Vascular Biology Unit, Evans Memorial Department of Clinical Research and Whitaker Cardiovascular Institute, \\ Boston University Medical Center, Boston, Massachusetts 02118
}

\begin{abstract}
The effects of platelet-derived growth factor (PDGF) were studied in isolated rings of rat aorta contracted submaximally to phenylephrine. The BB isoform of PDGF elicited relaxation in rings with endothelium and further contraction in rings without endothelium. Both the endothelium-dependent relaxation and endothelium-independent contraction occurred at concentrations known to induce PDGF receptor-mediated responses in cultured cells. Furthermore, the relaxation was isoform specific. This conclusion is supported by the unique ability of PDGF-BB to induce endothelium-dependent relaxations, as well as by studies showing isoform specific, concentration-dependent desensitization of PDGF-BB relaxation. The relaxation induced by PDGF-BB was prevented by $\mathbf{N} \omega-$ nitro-L-arginine. It was also observed that endothelium-independent contractions to the $A B$ and $A A$ isoforms of PDGF were less than those to PDGF-BB. Contrary to the widely held view that PDGF receptors are not present on the endothelium of macrovessels, these studies provide evidence for an endothelium-dependent, nitric oxide mediated relaxation of rat aorta caused by PDGF via PDGF $\beta \beta$-receptors. (J. Clin. Invest. 89:878-882.) Key words: $\beta \beta$-receptor • endothelium • nitric oxide • plateletderived growth factor $\bullet$ rat aorta
\end{abstract}

\section{Introduction}

Platelet-derived growth factor (PDGF) ${ }^{1}$ is a peptide dimer known to exist in three isoforms $(\mathrm{BB}, \mathrm{AB}$, and $\mathrm{AA})$ that exerts its biological effects via three different receptor types $(\beta \beta, \alpha \beta$, and $\alpha \alpha$ ). It is a potent mitogen and has been implicated in the vascular response to injury and the pathogenesis of atherosclerosis and hypertension (1-3). These conditions are associated with cell proliferation and/or migration as well as abnormal vasomotor responses (1-7). PDGF is expressed in the walls of normal arteries under circumstances where cell turnover is low, suggesting that PDGF may have important nonmitogenic functions (8). Based on studies showing that PDGF causes endothelium-independent contractions of vascular strips in vitro,

Address correspondence to Richard A. Cohen, M.D., Vascular Biology Unit, E401 University Hospital, 88 E. Newton St., Boston, MA 02118.

Received for publication 8 August 1991 and in revised form 8 November 1991.

1. Abbreviations used in this paper: Ach, Acetylcholine; NNA, No-nitro-L-arginine; PDGF, platelet-derived growth factor; PE, phenylephrine; PSS, physiological salt solution.

J. Clin. Invest.

(C) The American Society for Clinical Investigation, Inc.

$0021-9738 / 92 / 03 / 0878 / 05 \$ 2.00$

Volume 89, March 1992, 878-882 it is thought that PDGF may have a role in the modulation of vascular tone (9).

Recent studies of cultured microvascular endothelial cells have shown that PDGF receptors are present on these cells and that treatment with PDGF results in phosphorylation of the PDGF receptor, increases in glucose and basic amino acid uptake and stimulation of DNA synthesis $(10,11)$. However, it is a widely held view that PDGF receptors are not present on the endothelium of large vessels (10-18). Also, it is not known if the interaction of PDGF with endothelial cell receptors is capable of eliciting changes in vascular tone. The purpose of this study was to determine possible endothelium-dependent vasoactive effects of PDGF in macrovessels and to characterize these responses.

\section{Methods}

Aortic rings. Descending thoracic aortae were excised from male Wistar rats (276-300 g) killed by exsanguination after anesthesia with ketamine $(80 \mathrm{mg} / \mathrm{kg}$ i.m.) and xylazine $(14 \mathrm{mg} / \mathrm{kg}$ i.m.) and anticoagulation with sodium heparin ( $100 \mathrm{U}$ i.v.). The aortae were cleaned of adherent connective tissue and cut into transverse rings $4 \mathrm{~mm}$ in length. The endothelium was removed from some rings by inserting small forceps into the lumen and gently rolling the ring on moistened filter paper (19).

Vasoactive effects of PDGF isoforms. To study possible changes of isometric circumferential force in response to PDGF, rings with and without endothelium were suspended from strain gauges in organ baths containing $7 \mathrm{ml}$ of physiological salt solution (PSS) of the following composition (mM): $\mathrm{NaCl} 118.3, \mathrm{KCl} 4.7, \mathrm{MgSO}_{4}$ 1.2, $\mathrm{KH}_{2} \mathrm{PO}_{4}$ 1.2, $\mathrm{CaCl}_{2} 2.5, \mathrm{NaHCO}_{3} 25, \mathrm{Na}_{2}$-EDTA 0.026, and glucose 11.0. Once mounted, the rings were maintained at $37^{\circ} \mathrm{C}$ and gassed with $95 \%$ $\mathrm{O}_{2} / 5 \% \mathrm{CO}_{2}$. The rings were stretched stepwise to a resting tension of 5 $\mathrm{g}$, allowed to equilibrate for $60 \mathrm{~min}$ and then contracted with the $\alpha$ adrenergic agonist phenylephrine (PE) to $\sim 40 \%$ of a maximal contraction to potassium $(120 \mathrm{mM})$ for all studies. Mechanical denudation of the endothelium had no significant effect on either maximal contractions to potassium $(120 \mathrm{mM})$, or the concentration of $\mathrm{PE}$ required to achieve $40 \%$ of the potassium contraction (data not shown). The effect of each PDGF isoform (4-1,200 pM) on stable PE-induced tone was then determined by increasing the organ chamber concentration of the isoform by cumulative half-log increments after a maximal response was reached to each increment. Following the PDGF concentration response, the rings were washed with three volumes of fresh PSS and allowed to equilibrate for $30 \mathrm{~min}$. The rings were once again contracted with $\mathrm{PE}$ and responses to cumulative concentrations of acetylcholine (Ach, $10^{-8}$ to $10^{-5} \mathrm{M}$ ) were obtained to assess the functional integrity of the endothelium (20). Rings that relaxed more than $75 \%$ of PE-induced tone in response to Ach were considered to have an intact endothelium. Rings that relaxed less than $10 \%$ of PE-induced tone to Ach were considered endothelium-denuded.

Effect of incubation with PDGF isoforms on $P D G F-B B$ relaxations. Endothelium-dependent relaxations caused by PDGF-BB were studied after incubation of rings mounted in organ baths under $5 \mathrm{~g}$ of tension for $3 \mathrm{~h}$ at $37^{\circ} \mathrm{C}$ in PSS containing: (a) no additions, (b) PDGF-BB (4, 
40, or $400 \mathrm{pM}$ ), (c) PDGF-AB (400 pM), or (d) PDGF-AA (400 pM). PSS was replaced once every hour during the incubation period with fresh PSS containing the same additions. After the incubation period, the chambers were washed out with three volumes of fresh PSS and allowed to equilibrate for $\sim 20 \mathrm{~min}$ in the absence of PDGF before beginning PDGF concentration response curves.

Effect of cyclooxygenase and nitric oxide inhibition on PDGF-BB relaxations. The effect of cyclooxygenase inhibition on the endothelium-dependent relaxation to PDGF-BB was studied by adding indomethacin $(1.0 \mu \mathrm{M})$ to the organ chamber $30 \mathrm{~min}$ before beginning the PDGF-BB concentration response. To test the ability of an inhibitor of nitric oxide synthesis to prevent relaxation to PDGF-BB, some rings were treated for $30 \mathrm{~min}$ with the arginine analog $\mathrm{N} \omega$-nitro-L-arginine (NNA, $30 \mu \mathrm{M}$ ). After the 30 min treatment, the PSS containing NNA was replaced with PSS that did not contain NNA. The treated rings were allowed to equilibrate for $\sim 20$ min before beginning the PDGF concentration response. To test the ability of L-arginine $(100 \mu \mathrm{M})$ to reverse the effects of NNA, this amino acid was added to the chamber for the 20-min equilibration period following NNA treatment and was present throughout the PDGF concentration response.

Drugs. Human recombinant PDGF was used throughout (PDGFBB and PDGF-AA from PeproTech Inc., Rocky Hill, NJ; PDGF-AB from Upstate Biotechnology, Inc., Lake Placid, NY). Concentrations of PDGF were calculated using a calculated average molecular weight of $25 \mathrm{kD}$ for all three PDGF isoforms. Acetylcholine chloride, L-phenylephrine hydrochloride, indomethacin, and $\mathrm{N} \omega$-nitro-L-arginine were obtained from Sigma Chemical Co. (St. Louis, MO).

Data analysis. All responses are expressed as a percentage of PE-induced tone. Data are presented as means \pm SEM. For all experiments, $n$ equals the number of rats from which rings of aorta were taken. Significant differences between concentration-response curves and responses induced by a single concentration of agonist were determined by the Student $t$ test for unpaired observations. Contractile concentration-response curves to PDGF were compared by analysis of variance. Results were considered statistically significant when $P<0.05$.

\section{Results}

Responses of rings with and without endothelium to PDGF. In rings denuded of endothelium, all three isoforms of PDGF caused slight contractions, although contractions caused by PDGF-BB $(n=4)$ were significantly larger than those elicited by either PDGF-AB $(n=6)$ or PDGF-AA $(n=5, P<0.01$; Fig. $1, a$ and $c$ ). The maximal degree of contraction caused by PDGF-BB $(1,200 \mathrm{pM})$ was modest, averaging $0.76 \pm 0.18 \mathrm{~g}$ or $13.6 \pm 2.8 \%$ of the maximal tone induced by depolarization with potassium ( $120 \mathrm{mM}, 5.4 \pm 0.4 \mathrm{~g}, n=4)$. In contrast, in rings that had an intact endothelium, PDGF-BB caused concentration-dependent relaxations (Fig. $1, b$ and $d$ ). At similar concentrations, PDGF-AB produced mild contractions and PDGF-AA had virtually no effect (Fig. $1 d$ ).

Effect of incubation with PDGF isoforms on PDGF-BB relaxations. Cumulative data showing the effects on PDGF-BB relaxation of $3 \mathrm{~h}$ incubation without or with each of the three PDGF isoforms are shown in Fig. $2 a$. Incubation in the absence of PDGF had no effect on PDGF-BB induced relaxations $(n=12$, Fig. 2 a). Incubation with 400 pM PDGF-BB completely blocked the endothelium-dependent relaxations to PDGF-BB ( $n=8$, Fig. $2 a$ ). Incubation with either PDGF-AB $(400 \mathrm{pM}, n=9$ ) or PDGF-AA ( $400 \mathrm{pM}, n=8$ ) had no signifcant effect on the subsequent PDGF-BB induced relaxations (Fig. $2 a$ ). Relaxations to the endothelium-dependent vasodilator, Ach, following the concentration-response to PDGF-BB a

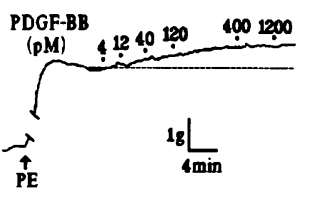

b
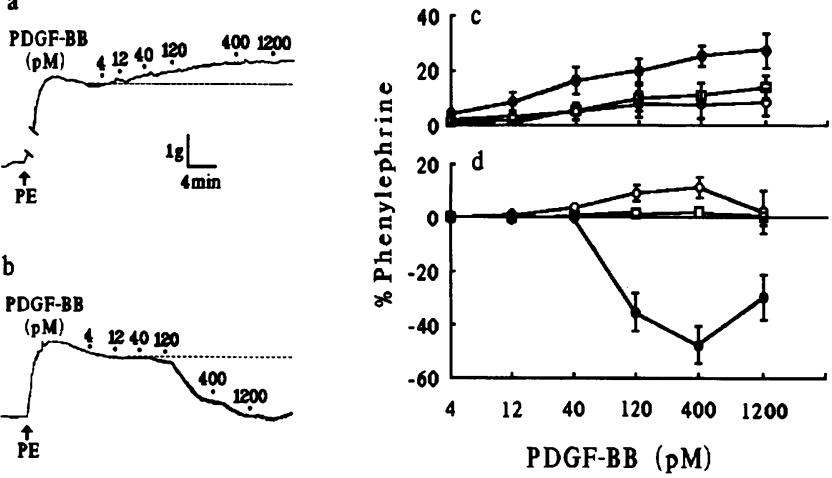

Figure 1. Representative tracings and cumulative data for responses to PDGF in rings of Wistar rat aorta with and without intact endothelium. Tracings show contractions $(a)$ and relaxations $(b)$ in response to increasing concentrations of PDGF-BB in rings without $(a)$ and with intact $(b)$ endothelium, respectively. For all studies, aortic rings were contracted with phenylephrine before beginning responses to PDGF. Cumulative data (c) for PDGF-BB $(\bullet, n=4)$, PDGF-AB $(0, n=6)$ and PDGF-AA ( $\square, n=5)$ in endothelium denuded rings showing that PDGF-BB caused significantly greater contractions than either PDGF-AB or PDGF-AA $(P<0.01)$. Cumulative results $(d)$ obtained from rings with endothelium showing responses to PDGF$\mathrm{AB}(0, n=7)$, PDGF-AA (ㅁ, $n=7)$, and PDGF-BB $(\bullet, n=23)$. Data are expressed as a percent of the phenylephrine contraction. Vertical bars $(c$ and $d)$ indicate the SEM.

were not affected by any of the incubation conditions (Table I). Incubation with increasing concentrations of PDGF-BB (4 $\mathrm{pM}, n=5 ; 40 \mathrm{pM}, n=5 ; 400 \mathrm{pM}, n=4)$ produced concentra-

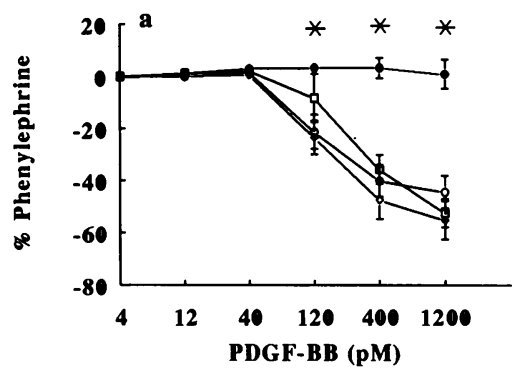

Figure 2. Cumulative data from studies incubating aortic rings having an intact endothelium with each of the three PDGF isoforms. (a) Results showing the inhibitory effect of $3 \mathrm{~h}$ incubation with PDGF$\mathrm{BB}(\bullet, n=8,400 \mathrm{pM})$ on subsequent PDGF$B B$ responses. Neither PDGF-AB (O, $n=9)$, nor PDGF-AA (, $n$

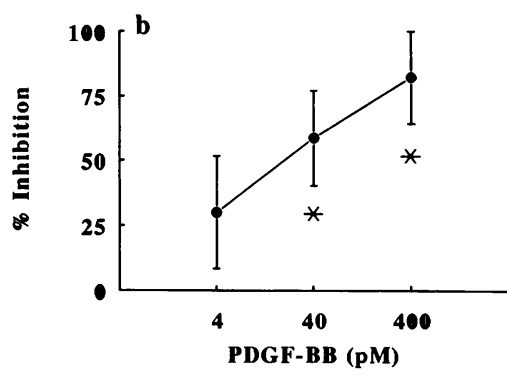

$=8)$ at a concentration of $400 \mathrm{pM}$ had a significant effect when compared to rings incubated without PDGF $(\diamond, n$ $=12$ ). Significant inhibition is shown by asterisks. (b) Cumulative data showing concentration-dependent inhibition of relaxations to PDGF-BB following a

$3 \mathrm{~h}$ incubation at $37^{\circ} \mathrm{C}$ with PDGF-BB ( $4 \mathrm{pM}, n=5 ; 40 \mathrm{pM}, n=5$; $400 \mathrm{pM}, n=4)$. Significant inhibition $(P<0.05)$, shown by asterisks, resulted from incubation with 40 and 400 pM PDGF-BB. Data are expressed as percent inhibition of responses to PDGF-BB $120 \mathrm{pM}$ in rings with endothelium incubated with PDGF-BB as compared to the response of rings simultaneously incubated without PDGF. Vertical bars indicate the SEM. 
Table I. Effect of 3 Incubation with PDGF Isoforms on Relaxations to Acetylcholine

\begin{tabular}{lrllr}
\hline & \multicolumn{4}{c}{ Acetylcholine concentration } \\
\cline { 2 - 4 } & \multicolumn{1}{c}{$0.01 \mu \mathrm{M}$} & \multicolumn{1}{c}{$0.1 \mu \mathrm{M}$} & \multicolumn{1}{c}{$1.0 \mu \mathrm{M}$} & $n$ \\
\hline Control & $-16.83 \pm 4.44$ & $-63.43 \pm 7.94$ & $-85.47 \pm 6.15$ & 11 \\
PDGF-BB & $-7.61 \pm 3.46$ & $-48.90 \pm 8.43$ & $-78.57 \pm 7.98$ & 8 \\
PDGF-AB & $-23.25 \pm 7.86$ & $-70.47 \pm 10.08$ & $-89.70 \pm 6.14$ & 6 \\
PDGF-AA & $-12.40 \pm 3.83$ & $-57.00 \pm 6.13$ & $-86.63 \pm 2.90$ & 8 \\
& & & & \\
\hline
\end{tabular}

Values are means \pm SEM. Data are relaxations expressed as a percent of the PE contraction. Following the incubation studies described in Fig. $2 a$, rings were contracted with PE and relaxations to cumulative concentrations of acetylcholine recorded. Statistical comparison showed that none of the incubation conditions had a significant effect on relaxations to acetylcholine as compared to the incubated control. $n$ indicates the number of incubated aortae in which acetylcholine responses were studied.

tion-dependent inhibition of relaxations to PDGF-BB (120 pM; Fig. $2 b$ ).

Effect of inhibitors on relaxations to PDGF-BB. PDGF-BB induced relaxations were not significantly affected by indomethacin (1.0 $\mu \mathrm{M}, n=5$; data not shown). However, treatment with NNA $(30 \mu \mathrm{M})$, a stereospecific inhibitor of nitric oxide synthase, blocked the endothelium-dependent relaxation to PDGF-BB ( $n=13$, Fig. 3). Relaxations to sodium nitroprusside $(1.0 \mu \mathrm{M})$ were not different in rings treated with NNA as compared to control $(-100.58 \pm 1.65$ vs. $-97.17 \pm 1.73$, respectively, expressed as a percent of phenylephrine contraction). Following treatment with NNA, PDGF-BB caused contractions that were comparable to those obtained in response to PDGF-BB in rings without endothelium $(0.77 \pm 0.24 \mathrm{~g})$. The addition of L-arginine $(100 \mu \mathrm{M})$ following NNA treatment, $\sim 20$ min before the PDGF concentration response, significantly reversed the inhibitory effects of NNA $(n=7$, Fig. 3$)$.

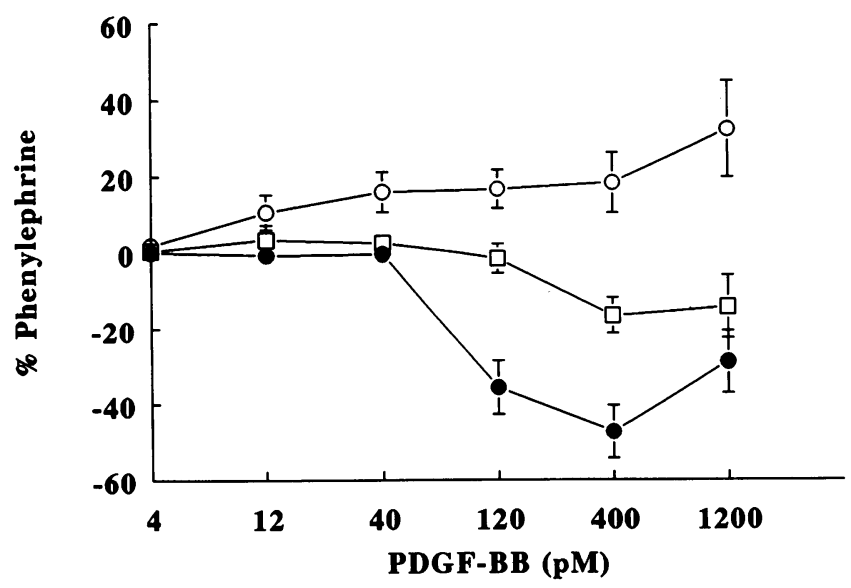

Figure 3. Cumulative data showing that $30 \mathrm{~min}$ incubation of aortic rings with endothelium in $30 \mu \mathrm{M}$ NNA $(0, n=13)$ prevented the PDGF-BB induced relaxations observed in control rings $(\bullet, n=23)$. The inhibitory effect of NNA was significantly reversed by treatment with $100 \mu \mathrm{M}$ L-arginine ( $\square, n=7, P<0.05$ ). Vertical bars indicate the SEM.

\section{Discussion}

Berk et al., in the original report describing a vasoactive effect of PDGF, found that PDGF-induced contractions are unaffected by removal of the endothelium (9). This, in addition to their finding that incubation with PDGF had no effect on Achinduced relaxation of intact rat aortic strips, led them to the conclusion that PDGF exerts its vasoactive effects in an endothelium-independent manner. This conclusion is in agreement with studies of cultured cells that show macrovascular endothelial cells do not respond to PDGF, presumably because they lack PDGF receptors (10-18). The observation of endothelium-independent responses to PDGF-AB and AA in the present study lends support to this view. However, the demonstration of endothelium-dependent relaxations in response to PDGF-BB suggests that PDGF can act on the endothelium of large vessels, but that such interactions may be isoform specific. The isoform-specific endothelium-mediated effects of PDGF would not have been apparent in the studies by Berk and colleagues as the PDGF preparation used was a mixture of PDGF isoforms $(9,20)$. Block et al. have also described endothelium-independent contractions to PDGF, but these and other studies were conducted without first inducing active vascular tone $(9,21,22)$. These differences from the present study could account for the absence of an effect of the endothelium in earlier reports. A third possibility is that the agonist used to constrict the vessels in the present study stimulated the expression of endothelial cell PDGF receptors, a phenomenon that has been described in cultured vascular smooth muscle cells (23). Even this explanation presumes a capacity for de novo synthesis of PDGF receptors by the aortic endothelium. It is however, still an unsatisfactory explanation of the endothelium-dependent response described, given the short period of time between exposure to the contractile agonist and the PDGF concentration response. A less likely possibility is that in the present study PDGF-BB caused endothelium-dependent relaxations indirectly by first acting on PDGF $\beta \beta$-receptors in the underlying smooth muscle. Thus, the discovery of an isoform-specific, endothelium-dependent relaxation of rat aortic rings to PDGF-BB is the first evidence for an endothelium-mediated effect of PDGF on vascular smooth muscle.

PDGF-BB initiated endothelium-dependent relaxations at concentrations approximately 10 -fold higher than those required for vasoconstriction (Fig. 1). It has been noted by others that PDGF induces contractions of vascular strips at concentrations lower than those required for stimulating either maximal increases in intracellular calcium or growth $(9,21)$. In the present study, endothelium-dependent relaxation to PDGFBB occurred in a concentration range that includes the $K_{\mathrm{d}}$ of $140 \mathrm{pM}$ for PDGF receptors and the $\mathrm{EC}_{50}$ of $300 \mathrm{pM}$ for halfmaximal PDGF receptor autophosphorylation reported by Beitz et al. in cultured microvascular endothelial cells (12). Thus, the concentrations at which relaxations to PDGF-BB occurred are consistent with those known to elicit PDGF receptor-mediated responses in cultured microvascular endothelium.

It is known that PDGF interacts with PDGF receptors in an isoform-specific manner such that $\alpha \alpha$-receptors bind all three PDGF isoforms, $\alpha \beta$-receptors bind only PDGF-AB and PDGF-BB, and $\beta \beta$-receptors bind only PDGF-BB (24). PDGF$\mathrm{BB}$ was the only isoform to cause endothelium-dependent relaxations. This result can be interpreted to indicate that the 
relaxations were due to the interaction of PDGF-BB with endothelial cell $\beta \beta$-receptors.

The isoform-specificity of the endothelium-dependent relaxation to PDGF is further supported by incubation studies conducted with each PDGF isoform. Reports from studies of cultured cells have shown that incubation with PDGF under conditions similar to the ones employed in this study is sufficient to cause isoform-specific downregulation of PDGF receptors and a consequent reduction in PDGF binding (24-26). PDGF-BB was the only isoform to inhibit the endothelium-dependent relaxation to PDGF following incubation, consistent with an isoform specific downregulation of $\beta \beta$-receptors. Failure of incubation with either PDGF-AB or PDGF-AA to block the endothelium-dependent relaxation is presumably due to the inability of these isoforms to bind at $\beta \beta$-receptors. The inhibitory effect of incubation with PDGF-BB was concentration-dependent, and even a concentration of PDGF-BB that did not elicit relaxation $(40 \mathrm{pM})$ was able to significantly inhibit subsequent endothelium-dependent relaxation to PDGFBB. Furthermore, inhibition occurred without affecting endothelium-dependent responses to Ach. These results suggest that the effects of incubation with PDGF-BB were specific for PDGF-induced relaxation and not due to nonspecific desensitization of the endothelium or the underlying smooth muscle. These findings are consistent with inhibition that results from PDGF receptor downregulation. Also, the ready availability of PDGF from platelets and the ability of vascular smooth muscle and endothelial cells to produce and secrete PDGF $(2,21)$ suggests that the ability of the endothelium to respond to PDGF and exert effects on the smooth muscle may be subject to autocrine or paracrine modulation.

Endothelium-dependent vasodilators act by causing the release of vasoactive prostanoids (27) or the endothelium-derived relaxing factor, nitric oxide (28). The apparent insensitivity of the endothelium-dependent relaxation to the cyclooxygenase inhibitor indomethacin indicates that the relaxation to PDGF-BB was probably not due to endothelium-derived prostanoids. It is now recognized that endothelium-derived nitric oxide, an activator of soluble guanylate cyclase, is synthesized from L-arginine and that certain L-arginine analogs act as competitive inhibitors of nitric oxide synthesis (28-31). In this study, the ability of the arginine analog NNA to completely block the relaxation of PDGF-BB and unmask contractions is comparable to those seen in rings denuded of endothelium, and strongly suggests that the endothelium-dependent relaxation to PDGF-BB is the result of nitric oxide release. The observation that relaxations to sodium nitroprusside were not affected by NNA, coupled with the partial restoration of relaxation to PDGF-BB by supplementation of NNA treated rings with L-arginine, indicate the specificity of NNA for nitric oxide synthase (30). That L-arginine was capable of only partially restoring the endothelium-dependent relaxation is not unexpected, given the potency of this particular arginine analog (31). In separate studies, PDGF-BB also caused endotheliumdependent, NNA-inhibitable, relaxations of porcine coronary artery, but not of rabbit thoracic aorta (data not shown).

This study demonstrates that PDGF-BB causes an isoform specific, endothelium-dependent relaxation of isolated rat aorta. Further evidence showing that arginine-derived nitric oxide mediates this relaxation suggests the presence of previously unrecognized, functional PDGF $\beta \beta$-receptors on macrovascular endothelium. PDGF stimulation of endothelial cells may be responsible for the routine replacement and/or repair of damaged or aging vascular endothelial cells. Alternatively, the importance of PDGF- $\beta \beta$ receptor-mediated effects on endothelial cells may rest in their ability to stimulate nitric oxide release. The vascular endothelium may have an important role in regulating the growth of the underlying smooth muscle (32), possibly by releasing nitric oxide (33) that may serve to counteract the direct proliferative effect that PDGF has on the smooth muscle. The loss of this internal control early in vascular diseases featuring abnormal endothelium-dependent responses may help explain the subsequent proliferation of smooth muscle cells that often occurs as a part of the vascular response and which characterizes the pathogenesis of these diseases.

\section{Acknowledgments}

L. Cunningham is supported by an American Heart Association Medical Student Research Fellowship. R. Cohen is supported by an American Heart Association Established Investigator Award. This research was supported by National Heart, Lung, and Blood Institute grants HL-47124, HL-31607, and HL-38731.

\section{References}

1. Majesky, M. W., M. A. Reidy, D. F. Bowen-Pope, C. E. Hart, J. N. Wilcox, and S. M. Schwartz. 1990. PDGF ligand and receptor gene expression during repair of arterial injury. J. Cell Biol. 111:2149-2158.

2. Ross, R., E. W. Raines, and D. F. Bowen-Pope. 1986. The biology of platelet-derived growth factor. Cell. 46:155-169.

3. Schwartz, S. M., R. L. Heimark, and M. W. Majesky. 1990. Developmental mechanisms underlying pathology of arteries. Physiol. Rev. 70:1177-1209.

4. Alosachie, I., and T. Godfraind. 1988. The modulatory role of vascular endothelium in the interaction of agonists and antagonists with $\alpha$-adrenoceptors in the rat aorta. Br. J. Pharmacol. 95:619-629.

5. Bossalier, C., G. B. Habib, H. Yamamoto, C. Williams, S. Wells, and P. D. Henry. 1987. Impaired muscarinic endothelium-dependent relaxation and cyclic guanosine 5'-monophosphate formation in atherosclerotic human coronary artery and rabbit aorta. J. Clin. Invest. 79:170-174.

6. Cohen, R. A., K. M. Zitnay, C. C. Haudenschild, and L. D. Cunningham. 1988. Loss of selective endothelial cell vasoactive functions caused by hypercholesterolemia in pig coronary arteries. Circ. Res. 63:903-910.

7. Otsuka, Y., A. DiPiero, E. Hirt, B. Brennaman, and W. Lockette. 1988. Vascular relaxation and CGMP in hypertension. Am. J. Physiol. 254:H163H169.

8. Barrett, T. B., and E. P. Benditt. 1988. Platelet-derived growth factor gene expression in human atherosclerotic plaques and normal artery wall. Proc. Natl. Acad. Sci. USA. 85:2810-2814.

9. Berk, B. C., R. W. Alexander, T. A. Brock, M. A. Gimbrone, Jr., and R. C. Webb. 1986. Vasoconstriction: A new activity for platelet-derived growth factor. Science (Wash. DC). 232:87-90.

10. Bar, R. S., M. Boes, B. A. Booth, B. L. Dake, S. Henley, and M. N. Hart. 1989. The effects of platelet-derived growth factor in cultured microvessel endothelial cells. Endocrinology. 124:1841-1848.

11. Beitz, J. G., I. Kim, P. Calabresi, and A. R. Frackelton, Jr. 1991. Human microvascular endothelial cells express receptors for platelet-derived growth factor. Proc. Natl. Acad. Sci. USA. 88:2021-2025.

12. Haudenschild, C. C., D. Zahniser, J. Folkman, and M. Klagsbrun. 1976. Human vascular endothelial cells in culture. Lack of response to serum growth factors. Exp. Cell Res. 98:175-183.

13. Wall, R. T., L. A. Harker, L. J. Quadracci, and G. E. Striker. 1978. Factors influencing endothelial cell proliferation in vitro. J. Cell. Physiol. 96:203-214.

14. Davies, P. F., and R. Ross. 1978. Mediation of pinocytosis in cultured arterial smooth muscle and endothelial cells by platelet-derived growth factor. $J$. Cell Biol. 79:663-671.

15. Heldin, C., B. Westermark, and A.. Wasteson. 1981. Specific receptors for platelet-derived growth factor on cells derived from connective tissue and glia. Proc. Natl. Acad. Sci. USA. 78:3664-3668.

16. Bowen-Pope, D. F., and R. Ross. 1982. Platelet-derived growth factor. II. Specific binding to cultured cells. J. Biol. Chem. 257:5161-5171.

17. Kazlauskas, A., and P. E. DiCorleto. 1985. Cultured endothelial cells do not respond to a platelet-derived growth-factor-like protein in an autocrine manner. Biochim. Biophys. Acta. 846:405-412. 
18. Ristimaki, A., O. Ylikorkala, and L. Viinikka. 1990. Effect of growth factors on human vascular endothelial cell prostacyclin production. Arteriosclerosis. 10:653-657.

19. Furchgott, R. F. 1984. The role of endothelium in the responses of vascular smooth muscle to drugs. Annu. Rev. Pharmacol. Toxicol. 24:175-197.

20. Bowen-Pope, D. F., C. E. Hart, and R. A. Seifert. 1989. Sera and conditioned media contain different isoforms of platelet-derived growth factor (PDGF) which bond to different classes of PDGF receptor. J. Biol. Chem. 264:2502-2508.

21. Block, L. H., L. R. Emmons, E. Vogt, A. Sachinidis, W. Vetter, and J. Hoppe. 1989. $\mathrm{Ca}^{2+}$-channel blockers inhibit the action of recombinant plateletderived growth factor in vascular smooth muscle cells. Proc. Natl. Acad. Sci. USA 86:2388-2392.

22. Sachinidis, A., R. Locher, J. Hoppe, and W. Vetter. 1990. The plateletderived growth factor isomers, PDGF-AA, PDGF-AB and PDGF-BB, induce contraction of vascular smooth muscle cells by different intracellular mechanisms. FEBS (Fed. Eur. Biochem. Soc.) 275:95-98.

23. Bobik, A., S. Grinpukel, P. J. Little, A. Grooms, and G. Jackman. 1990 Angiotensin II and noradrenaline increase PDGF-BB receptors and potentiate PDGF-BB stimulated DNA synthesis in vascular smooth muscle. Biochem. Biophys. Res. Commun. 166:580-588.

24. Seifert, R. A., C. E. Hart, P. E. Phillips, J. W. Forstrom, R. Ross, M. J. Murray, and D. F. Bowen-Pope. 1989. Two different subunits associate to create isoform-specific platelet-derived growth factor receptors. J. Biol. Chem 264:8771-8778.
25. Heldin, C. H., A. Wasteson, and B. Westermark. 1982. Interaction of platelet-derived growth factor with its fibroblast receptor. J. Biol. Chem 257:4216-4221.

26. Hart, C. E., J. W. Forstrom, J. D. Kelly, R. A. Seifert, R. A. Smith, R. Ross, M. J. Murray, and D. F. Bowen-Pope. 1988. Two classes of PDGF recepto recognize different isoforms of PDGF. Science (Wash. DC). 240:1529-1531.

27. Moncada, S., and J. Vane. 1979. Pharmacology and the endogenous roles of prostaglandin endoperoxides, thromboxane $\mathrm{A}_{2}$ and prostacyclin. Pharmacol. Rev. 30:292-331.

28. Palmer, R. M. J., A. G. Ferrige, and S. Moncada. 1987. Nitric oxide release accounts for the biological activity of endothelium-derived relaxing factor Nature (Lond.). 327:524-526.

29. Palmer, R. M. J., D. S. Ashton, and S. Moncada. 1988. Vascular endothelial cells synthesize nitric oxide from L-arginine. Nature (Lond.). 333:664-666.

30. Moncada, S., M. J. Palmer, and E. A. Higgs. 1989. Biosynthesis of nitric oxide from $\mathrm{L}$-arginine. A pathway for the regulation of cell function and communication. Biochem. Pharmacol. 38:1709-1715.

31. Ishii, K., B. Chang, J. F. Kerwin, Jr., Z. Huang, and F. Murad. 1990. $\mathrm{N} \omega$-Nitro-L-arginine: a potent inhibitor of endothelium-derived relaxing factor formation. Eur. J. Pharmacol. 176:219-223.

32. De Mey, J. G. R., E. H. Dijkstra, and J. J. F. Vrijdag. 1991. Endothelium reduces DNA synthesis in isolated arteries. Am. J. Physiol. 260:H1128-H1134.

33. Garg, U.C., and A. Hassid. 1989. Nitric oxide-generating vasodilators and 8-bromo-cyclic guanosine monophosphate inhibit mitogenesis and proliferation of cultured rat vascular smooth muscle cells. J. Clin. Invest. 83:1774-1777. 